\title{
Evaluation of a Local Fault Detection Algorithm for HVDC Systems
}

\author{
M.J. Perez-Molina ${ }^{1}$, P. Eguia-Lopez ${ }^{1}$, D.M. Larruskain-Eskobal ${ }^{1}$, M. Santos-Mugica ${ }^{2}$ and R. \\ Rodriguez-Sanchez ${ }^{2}$ \\ ${ }^{1}$ Department of Electrical Engineering, Faculty of Engineering of Bilbao, Universidad del País Vasco UPV/EHU \\ Alameda Urquijo s/n, 48013 Bilbao (Spain) \\ Phone/Fax number: +0034 986 812685, e-mail: mariajose.perez@ehu.eus \\ ${ }^{2}$ Energy Unit, Tecnalia \\ Parque Tecnológico de Vizcaya, Edificio 700, 48160 Derio (Spain) \\ Phone/Fax number: +0034 986812685
}

\begin{abstract}
A great increase in the amount of energy generated from clean and renewable sources integrated in the electric power system is expected worldwide in the coming years. High Voltage Direct Current (HVDC) systems are seen as a promising alternative to the traditional Alternating Current (AC) systems for the expansion of the electric power system. However, to achieve this vision, there are some remaining challenges regarding HVDC systems which need to be solved. One of the main challenges is related to fault detection and location in HVDC grids. This paper reviews the main protection algorithms available and presents the evaluation of a local fault detection algorithm for DC faults in a multi-terminal Voltage Source Conversion (VSC) based HVDC grid. The paper analyses the influence of the DC voltage sampling frequency and the cable length in the performance of the algorithm.
\end{abstract}

\section{Key words}

DC faults, fault detection, local protection system, multiterminal, VSC-HVDC grid, protection algorithm.

\section{Introduction}

Historically, power systems have been mostly based in AC systems; consequently, DC systems are a minor part in the worldwide system.

Nowadays, however, HVDC technology offers great characteristics. Accordingly, HVDC systems are forecasted as the most promising solution for future expansions of the grid, new interconnections between nations or the connection of large power plants located in remote areas.

Some of these advantages over the AC option are the lesser number of electrical conductors needed to transmit the same amount of power (two poles in DC, three poles in AC) and the lower power losses in long distance power links.
These characteristics make HVDC systems a cheaper solution. The relationship between costs and transmission distance for HVDC and HVAC technologies is shown in Fig. 1.

Despite that, there are still unresolved technical issues in the DC side regarding fault detection and clearance. Due to the reduced line resistance of DC electrical conductors, two critical conditions take place during fault conditions; the current increases very fast and the propagation of the voltage drop is large and system wide. The former implies a major problem since overcurrents can cause important damages in the power electronic components of the converters; even their destruction [1]. This is especially important in the IGBTs of Voltage Source Converters (VSC). To avoid the damage, in the case of half-bridge converters, an internal protection trips the IGBTs. This way, the converter becomes an uncontrolled diode bridge and enables a path for the fault current and its propagation to the AC side of the system.

Because of this, very fast protection systems are needed. They must operate in a very short time; which is assumed to be in the order of a few milliseconds (around $10 \mathrm{~ms}$ [2]). As a result, very fast fault detection and location algorithms are needed.

Due to all this, the same protection algorithms used in AC systems cannot be used in HVDC systems without proper adaptations, but they should fulfil the same requirements: reliability, speed, economy, selectivity and sensitivity [3][4]. They should detect, locate and clear faults as fast as possible, disconnecting only the faulty part of the grid, keeping the healthy parts of the grid in operation. The latter statement is even more critical in multi-terminal and meshed HVDC grids. 


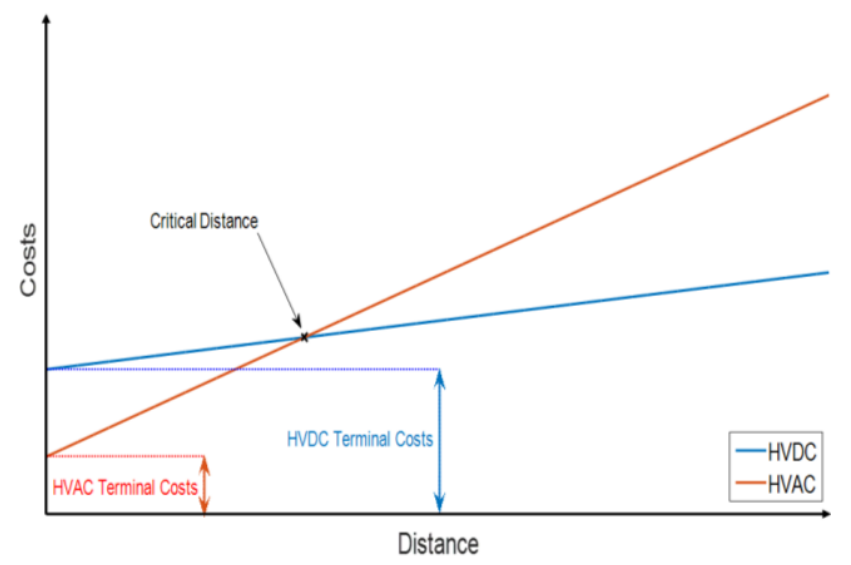

Fig. 1. Cost comparison of HVAC and HVDC technologies.

Fast HVDC circuit breakers (CB) are needed as well. Their response time must be as short as possible to prevent the damaging effects or even the destruction of the power electronic components of the converters when the current is higher than the withstand limits [3]. In addition, HVDC CBs must fulfil two functions which are not required for AC CBs. The first one is to produce a current zero, in order to assist the current interruption process since it does not cross zero naturally as in AC systems. The second function is to dissipate the energy stored in the system due to the fault condition [5].

The abovementioned functions significantly affect the behaviour of the HVDC $\mathrm{CB}$, which is a critical device in the reliability of HVDC grids. Likewise, its behaviour is determined by its current interruption capability and its operation speed [6].

Apart from that, for longer response times of the fault detection algorithm, an increased current interruption capability is needed. Moreover, the HVDC CBs will need to dissipate a greater energy value, hence increasing the costs. Thus, it is critical to shorten the response time of the fault detection algorithms.

Furthermore, the rate of rise of the current can be limited adjusting the value of the inductive terminations of the cables (DC reactors located at both ends of a cable). However, the size of this component affects not only the cost of the DC grid but also the stability [7].

In this paper, HVDC detection and location algorithms are reviewed. Afterwards, a fault detection algorithm based on local measurements is analysed and tested in a multiterminal HVDC system. Finally, the sensitivity of the algorithm to the DC voltage sampling frequency and cable length are analysed.

\section{HVDC detection and location algorithms}

As mentioned previously, detection and location algorithms are a very important part of the protection system. They need to be especially fast to help fulfilling the requirements of speed of HVDC protection systems. A fault should be detected, located and cleared as fast as possible, in the order of 10 milliseconds [2] to prevent, in the case of VSC-based systems, damage in the power electronic components and voltage collapse.

Detection and location algorithms can be classified into direct-measurement-based algorithms and signalprocessing-based algorithms. As example of directmeasurement-based algorithms there are the overcurrent, differential current and distance protection algorithms. As example of the signal-processing-based ones there is the travelling wave algorithm [3], [8].

Hereafter, some of these algorithms are described.

\section{A. Traveling wave algorithms}

When a fault occurs on a line, the fault wave is propagated from the fault point to the rest of the system. Different characteristics of the fault wave are used by protection systems that are based on this method for fault detection, location and discrimination.

Traveling wave algorithms can be classified in singleended or multi-ended (synchronized) if the measurements they use are obtained at only one end or at both ends of the protected transmission line respectively [9].

Traveling wave algorithms have generally fast response and high accuracy and its results are not easily affected by factors like bus configuration, fault type, loading conditions or system parameters.

However, they present some technical inconveniences such as the difficulty in the detection of the wave-front due to interference of signals and its dependence on the sampling frequency value [3], [10]-[12].

\section{B. Current differential algorithms}

These algorithms are based on measuring the current at both line ends and comparing the difference between the two measurements with a threshold value. The protection trips if the difference is greater than the threshold [4], [8].

Ideally, if the two current measurements are equal, there is no fault on the line, but if there is a difference between them, there is a fault on the line. In practice, a threshold value different than zero is used to account for measurement errors and other effects.

As measurements are taken at both ends of the line, it presents high selectivity [4] but data must be synchronised and accurate line parameters along with high sampling rate and high communication speed is needed. For this reason, this algorithm is often costly [3], [13], [14]. Moreover, the delay time imposed by the communication system makes its response time slower, especially in very long cable length conditions. Thus, the communication delay time is a critical factor in the total fault clearance time [15]. For this matter, they are mostly used as part of a back-up protection system or to protect against high impedance fault conditions [16] 


\section{Current-based algorithms}

In this case, the DC current is locally measured. This measurement can be directly compared with a threshold value, tripping the protection if the current measurement overcomes a threshold or it can be mathematically processed using its derivative instead. Current derivative algorithms are usually named rate of change of the current (ROCOC) algorithms.

However, these algorithms present reduced selectivity [3], [8], since it is a single-ended algorithm and could mistake an external fault of a nearby short cable as an internal fault.

\section{Voltage-based algorithms}

Similarly, the DC voltage is locally measured and, once again, can be directly compared with a threshold or mathematically processed. In this case, the protection system will operate if the voltage is lower than a threshold value. Voltage derivative algorithms are known as rate of change of voltage (ROCOV) algorithms.

These algorithms are much faster than those current-based since the voltage drop is sharper than the increase of the current during fault conditions [17].

\section{E. Directional protection algorithms}

In these algorithms, the current direction at both ends of the line is the parameter that indicates if there is a fault on the line, since the current values change abruptly when a fault occurs on the line, which cannot occur during normal operation [18].

During a fault condition on the line, the current flow at one end of the line will be reversed, while, at the other end, it will continue in the same direction [18].

This algorithm presents better selectivity than the previous ones, since the direction of the fault current determines its operation. Nevertheless, it has the problem that some protections that are located near the fault may be tripped unnecessarily [3], [8].

\section{F. Distance protection algorithms}

These algorithms estimate the fault distance by calculating the impedance between the protection device and the fault point, using voltage and current measurements.

When applied to HVDC systems, the estimated fault distance may be inaccurate due to the abrupt frequency changes during the initial fault transients [3], [8].

\section{Overcurrent and undervoltage algorithm}

The proposed fault detection algorithm is based on local current and voltage measurements. The values of the DC current and voltage are compared with their respective threshold values.
For application in a multi-terminal HVDC grid, DC current and voltage measurements are taken in each end of all HVDC links and processed locally. Hence, this algorithm is part of a non-unit protection system; it is nontelecommunication based and only local measurements are used.

A fault is detected when one of the following conditions is satisfied:

- the value of the DC current is greater than the current threshold value

or

- $\quad$ three consecutive sample values of the DC voltage are lower than the voltage threshold value.

This overcurrent and undervoltage $(O \& U)$ algorithm is used due to its short fault detection time and fast operation speed. It presents great reliability as well due to using the current and voltage measurements separately; so, if one of these measuring loops presents a problem and cannot properly detect a fault, the system is not affected because the other one can continue detecting the fault independently.

By using three consecutive sample values in the undervoltage part of the algorithm, the performance of the protection system is improved, presenting a better selectivity in its operation. With just one sample value, transients could be detected as faults, resulting in a noncorrect trip of the HVDC circuit breaker.

It is important to highlight that the undervoltage detection algorithm is faster than the overcurrent detection algorithm since the voltage drop is much sharper than the increase of the current during fault conditions.

Finally, when one of the above-mention conditions is satisfied, a fault is detected; and a signal is sent to the HVDC CB in order to trip and clear the fault.

\section{Study case}

The HVDC grid model proposed in [19] has been used in the study case to evaluate the performance of the algorithm described in Section 3. The model is shown in Fig. 2.

The model includes a multi-terminal VSC-HVDC grid consisting of four half-bridge Modular Multi-level Converters (MMC). Three converters are similar, of 900 MVA rating, and the other one is larger, of 1200 MVA.

The converters are linked by five HVDC cables with hybrid HVDC circuit breakers located at each end. The operating time of the hybrid HVDC $\mathrm{CBs}$ is assumed to be 2 milliseconds. The cables present inductive terminations, i.e., inductive reactors located in series with the DC CBs at each end of the cables. $100 \mathrm{mH}$ reactors are used in order to limit the fast increase of the DC fault current. 
The model presents a symmetrical monopole configuration; it has the inconvenient that when one of the cables is out of service, the power supply is stopped.

To analyse the sensitivity and behaviour of this overcurrent and undervoltage algorithm a large amount of different fault conditions have been simulated, varying parameters as cable length, fault distance and DC voltage sampling frequency. The study has been performed using PSCAD software.

\section{A. Performance test}

The performance of the algorithm has been verified in the model through simulations. The algorithm accurately detects different fault types (pole-to-pole, positive-pole-toground and negative-pole-to-ground faults) located along the cable length.

To evaluate its performance, different fault conditions were simulated varying two parameters: cable length and DC voltage sampling frequency.

\section{1) Cable length}

To analyse the sensitivity of the O\&U algorithm to different cable length conditions, a series of simulations varying the cable length where performed.

A pole-to-pole fault was simulated; the fault point was located right in front of one of the relays at one end of the cable. A DC voltage sampling frequency of $5 \mathrm{kHz}$ was used.

From these simulations, it was extracted that the lowest fault duration time detected by the O\&U algorithm increases exponentially with the cable length, as shown in Fig. 3.

The results of the time needed for the O\&U algorithm to detect a fault in these conditions is shown in Table I. The DC currents interrupted by the hybrid circuit breakers are presented as well (Fig. 5).

It must be highlighted that for cable lengths of 100 and 150 $\mathrm{km}$, the closest relay to the fault point detected the fault with the overcurrent algorithm. In other conditions, the fault was detected by the undervoltage algorithm.

Meanwhile, in the case of the farthest relay to the fault point, for all cable condition, the fault was detected by the undervoltage algorithm.

\section{2) DC voltage sampling frequency}

In this section, the sensitivity of the algorithm performance to the DC voltage sampling frequency is analysed. A poleto-pole fault was simulated; the fault point was located right in front of one of the relays at one end of the cable.

It was verified that for higher DC voltage sampling frequency in the algorithm, the value of the lowest fault duration time detected by the algorithm is lower, and vice versa, as it is shown in Fig. 3.

The times needed by both relays of the cable to detect a fault are presented in Fig. 4. The closest relay to the fault point needs a remarkably shorter time to detect the fault. This time can be considered constant regardless of the cable length. However, the farthest relay to the fault point needs more time to detect the fault; its fault detection time increases proportionally with the cable length.

On the other hand, the $\mathrm{CB}$ of the closest relay to the fault point needs to interrupt a greater current than the $\mathrm{CB}$ of farthest relay, as it can be seen in Fig. 5.

Another conclusion extracted from the results of the simulations is that the fault detection time and the current interrupted by the CBs would be lower when the DC voltage sampling frequency is higher.

\section{Conclusion}

In this paper, considering the significant prospective of HVDC technology in future power transmission systems and the actual challenge in the protection of these systems, an undervoltage and overcurrent algorithm is proposed and evaluated. To detect a fault on the cable, the algorithm uses direct local DC current and voltage measurements and compares them with an overcurrent threshold and an undervoltage threshold, respectively. By using consecutive samples of the voltage instead of just one sample, the selectivity of the protection system is improved. This way, fast voltage transients are less likely to be mistaken as faults.

The reliability of the protection system is further improved by making the comparison of the DC current and voltage measurements independently. If one of the conditions (undervoltage or overcurrent) is satisfied, a fault is detected in the protected cable. Also, if one of the measuring loops gets damaged, the protection system can continue operating properly thanks to the other healthy loop.

This O\&U algorithm is tested for different fault conditions and with variations of different parameters, such as cable length and DC voltage sampling frequency.

It is concluded that the lowest fault duration time detected by the algorithm increases exponentially with the cable length. Regarding the time needed for the algorithm to detect a fault, with higher DC voltage sampling frequencies, the fault detection is faster, and as a result, the circuit breaker interrupts a lower fault current.

\section{Acknowledgement}

The authors thank the support from the Spanish Ministry of Economy, Industry and Competitiveness (project ENE2016-79145-R AEI/FEDER, UE) and GISEL research group IT1083-16), as well as from the University of the Basque Country UPV/EHU (research group funding PPG17/23). 


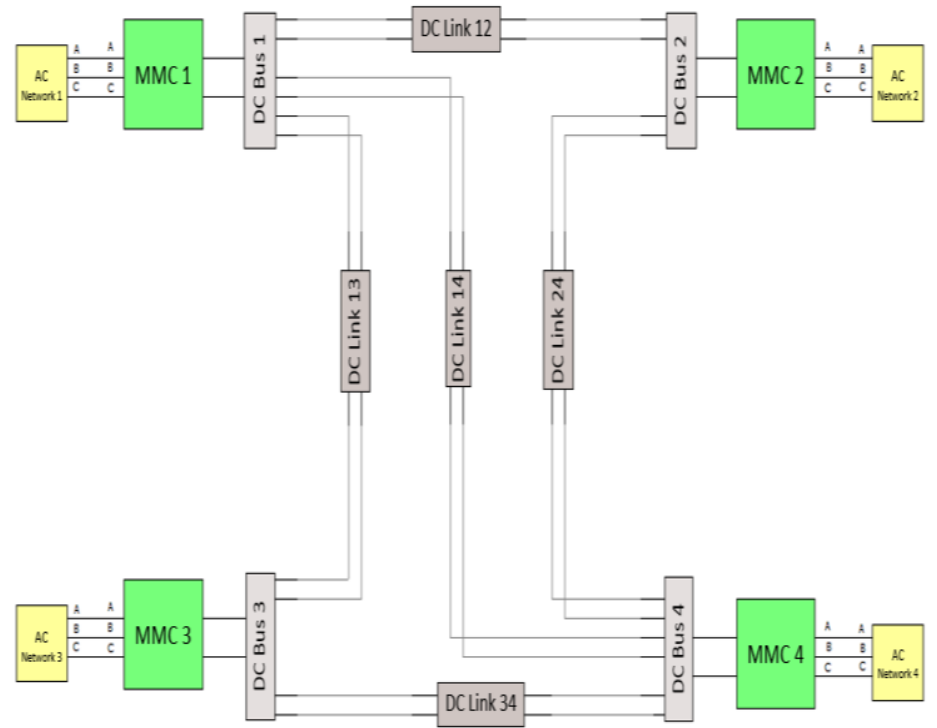

Table I. Fault detection time by the O\&U algorithm and value of the current interrupted by the circuit breaker regarding the cable length for the closest and farthest relay to the fault point.

\begin{tabular}{|c|c|c|c|c|}
\hline \multirow{2}{*}{$\begin{array}{c}\text { Cable } \\
\text { Length } \\
(\mathrm{km})\end{array}$} & \multicolumn{2}{|c|}{$\begin{array}{c}\text { Closest relay to the } \\
\text { fault point }\end{array}$} & \multicolumn{2}{c|}{$\begin{array}{c}\text { Farthest relay to the } \\
\text { fault point }\end{array}$} \\
\cline { 2 - 5 } & $\begin{array}{c}\text { Fault } \\
\text { Detection } \\
\text { Time }(\mathrm{ms})\end{array}$ & $\begin{array}{c}\text { Current } \\
(\mathrm{kA})\end{array}$ & $\begin{array}{c}\text { Fault } \\
\text { Detection } \\
\text { Time }(\mathrm{ms})\end{array}$ & $\begin{array}{c}\text { Current } \\
(\mathrm{kA})\end{array}$ \\
\hline 100 & 0,35 & 6,423 & 1 & 3,910 \\
\hline 150 & 0,4 & 6,468 & 1,3 & 4,443 \\
\hline 200 & 0,45 & 6,357 & 1,6 & 4,933 \\
\hline 250 & 0,45 & 6,379 & 1,95 & 4,949 \\
\hline 300 & 0,45 & 6,418 & 2,35 & 4,837 \\
\hline 350 & 0,45 & 6,357 & 2,8 & 4,806 \\
\hline 400 & 0,45 & 6,308 & 3,35 & 4,908 \\
\hline
\end{tabular}

Fig. 2. HVDC model schematic in PSCAD.

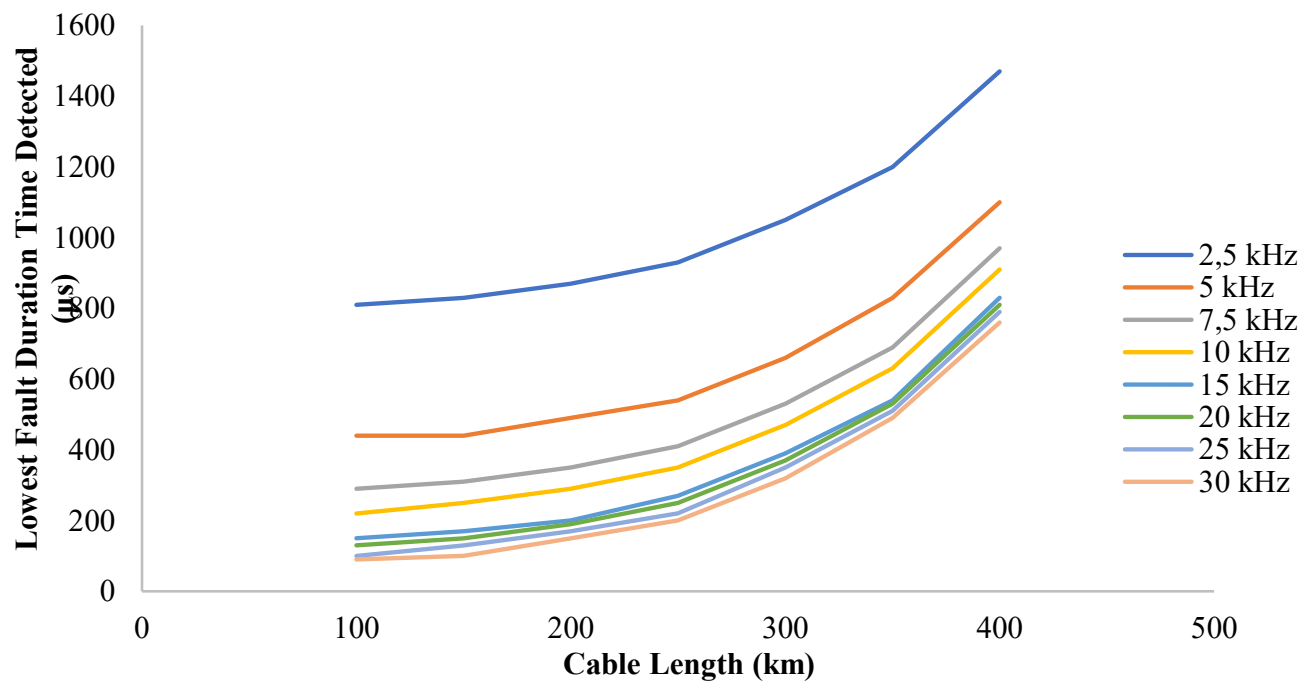

Fig. 3. Sensitivity of the algorithm as a function of cable length and DC voltage sampling frequency variations.

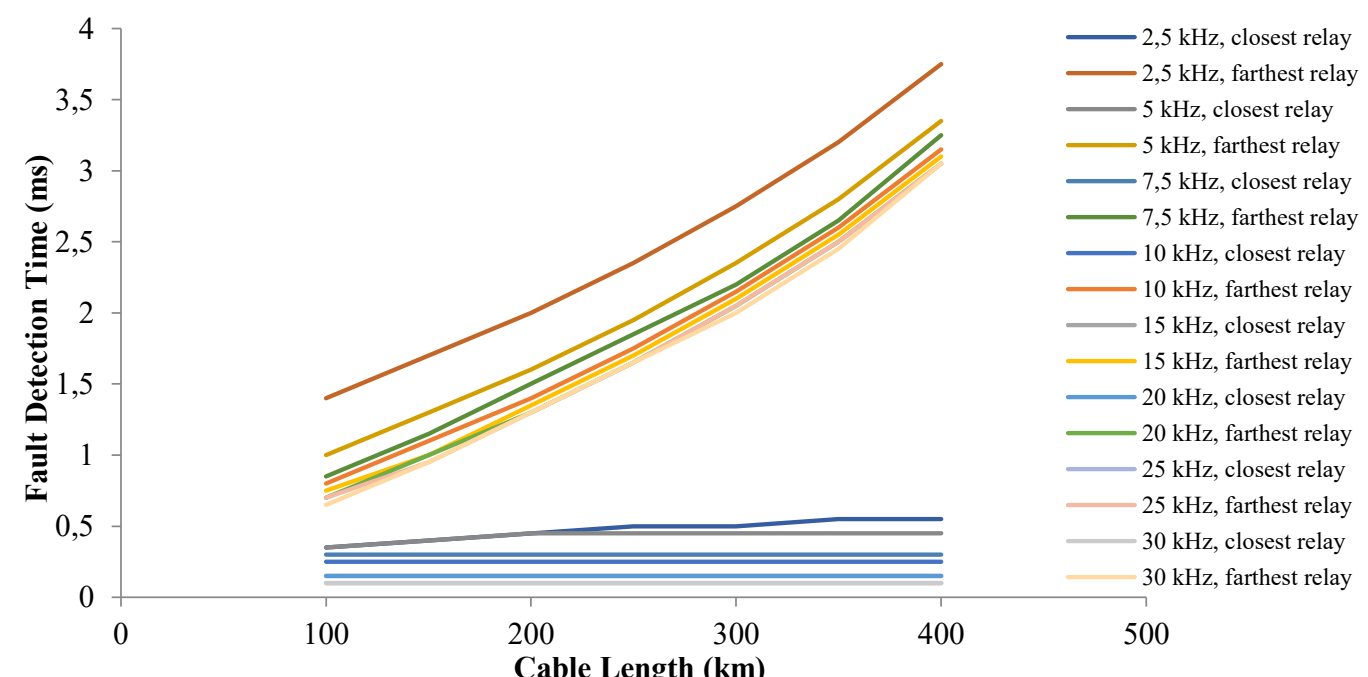

Fig. 4. Fault detection times of the closest and farthest relays to the fault point as a function of DC voltage sampling frequency and cable length. 


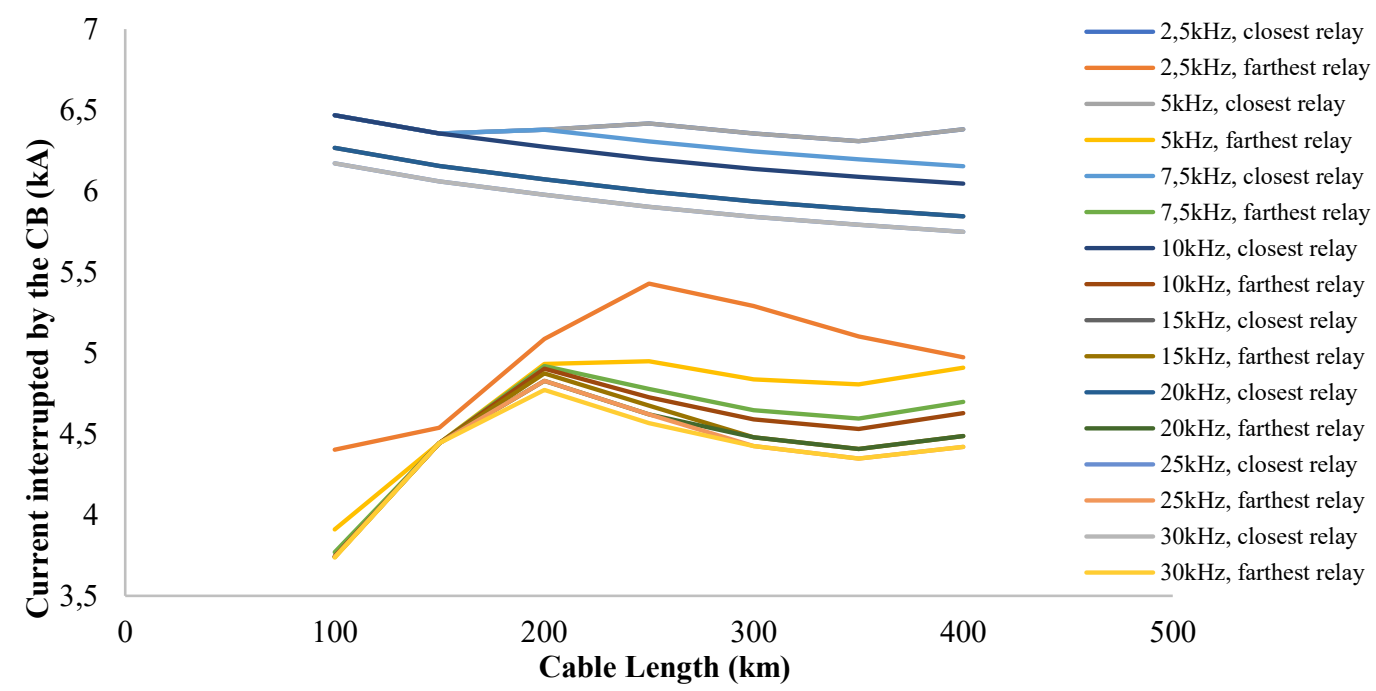

Fig. 5. Current interrupted by the circuit breakers of the closest and farthest relays to the fault point as a function of DC voltage sampling frequency and cable length.

\section{References}

[1] CIGRÉ WG B4.52, "HVDC Grid Feasibility Study," CIGRÉ, 2013. Available: https://e-cigre.org/publication/533-hvdc-gridfeasibility-study.

[2] J. Descloux et al, "HVDC Meshed Grid: Control and Protection of a Multi-terminal HVDC System," CIGRÉ Session Paris (Paper B4-308), pp. 10, 2012. Available: https://ecigre.org/publication/B4-308 2012-hvdc-meshed-grid-controland-protection-of-a-multi-terminal-hvdc-system.

[3] G. Buigues et al, "DC fault detection in VSC-based HVDC grids used for the integration of renewable energies," in 2015 International Conference on Clean Electrical Power (ICCEP), Taormina, Italy, 06 August 2015, pp. 666-673.

[4] A. K. Marten, C. Troitzsch and D. Westermann, "Nontelecommunication based DC line fault detection methodology for meshed HVDC grids," in 11th IET International Conference on $A C$ and DC Power Transmission, Birmingham, UK, 13 July 2015 , pp. 1-8.

[5] M. K. Bucher and C. M. Franck, "Fault Current Interruption in Multiterminal HVDC Networks," IEEE Transactions on Power Delivery, vol. 31, (1), pp. 87-95, 2016. Available: https://ieeexplore.ieee.org/stamp/stamp.jsp?tp=\&arnumber $=7131$ 567. DOI: 10.1109/TPWRD.2015.2448761.

[6] W. Leterme, "Communication-Less Protection Algorithms for Meshed VSC HVDC Cable Grids", KU Leuven, Leuven, Belgium, 2016.

[7] J. Häfner and B. Jacobson, "Proactive hybrid HVDC breakers - A key innovation for reliable HVDC grids," in The Electric Power System of the Future - Integrating Supergrids and Microgrids International Symposium, Bologna, Italy, 13-15 September 2011,

[8] R. E. Torres-Olguin and H. K. Høidalen, "Inverse time overcurrent protection scheme for fault location in multi-terminal HVDC," in 2015 IEEE Eindhoven PowerTech, Eindhoven, Netherlands, 03 September 2015, pp. 1-6.

[9] F. H. Magnago and A. Abur, "Fault location using wavelets," IEEE Transactions on Power Delivery, vol. 13, (4), pp. 14751480, $1998 . \quad$ Available: https://ieeexplore.ieee.org/stamp/stamp.jsp?tp=\&arnumber $=7148$ 08. DOI: $10.1109 / 61.714808$.

[10] G. Song et al, "Natural frequency based protection and fault location for VSC-HVDC transmission lines," in 2011
International Conference on Advanced Power System Automation and Protection, Beijing, China, 2011, pp. 177-182.

[11] Z. He et al, "Natural Frequency-Based Line Fault Location in HVDC Lines," IEEE Transactions on Power Delivery, vol. 29, (2), pp. 851-859, 2014. Available: https://ieeexplore.ieee.org/stamp/stamp.jsp?tp=\&arnumber $=6555$ 974. DOI: 10.1109/TPWRD.2013.2269769.

[12] H. Livani and C. Y. Evrenosoglu, "A single-ended fault location method for segmented HVDC transmission line," Electric Power Systems Research, vol. 107, pp. 190-198, February, 2014.

[13] G. Song et al, "A novel pilot protection principle for VSCHVDC cable lines based on fault component current," in 2012 Power Engineering and Automation Conference, Wuhan, China, 26 September 2013, pp. 1-4.

[14] S. P. Azad, W. Leterme and D. Van Hertem, "A DC grid primary protection algorithm based on current measurements," in 2015 17th European Conference on Power Electronics and Applications (EPE'15 ECCE-Europe), Geneva, Switzerland, 2015 , pp. 1-10.

[15] M. J. Pérez-Molina et al, "A comparison of non-unit and unit protection algorithms for HVDC grids," in AEIT HVDC International Conference 2019, Florence, Italy, 2019.

[16] D. Naidoo and N. M. Ijumba, "HVDC line protection for the proposed future HVDC systems," in 2004 International Conference on Power System Technology. PowerCon 2004. Singapore, Singapore, 05 July 2005, pp. 1332 Vol.2.

[17] A. Visakh and R. M. Shereef, "Protection of HVDC grids against temporary and permanent faults," in 2018 International Conference on Control, Power, Communication and Computing Technologies (ICCPCCT), Kannur, India, 13 December 2018, pp. 239-244.

[18] A. Samir et al, "A directional protection technique for MTDC networks," in 2015 4th International Conference on Electric Power and Energy Conversion Systems (EPECS), Sharjah, United Arab Emirates, 2015, pp. 1-6.

[19] W. Leterme et al, "A new HVDC grid test system for HVDC grid dynamics and protection studies in EMT-type software," in 11th IET International Conference on $A C$ and DC Power Transmission, 2015/01/01, Available: https://www.esat.kuleuven.be/electa/hvdcresearch/hvdc-test-grid. DOI: $10.1049 /$ cp.2015.0068. 